\title{
Propagation of aggrading sediment fronts in a laboratory flume
}

\author{
Alessio Radice, ${ }^{1, *}$ and Stefanía Unigarro Villota ${ }^{1}$ \\ ${ }^{1}$ Politecnico di Milano, Dept. of Civil and Environmental Engineering, Milan, Italy
}

\begin{abstract}
Channel bed aggradation due to sediment overloading was studied experimentally. A series of aggradation tests, with uniform lightweight sediment, were performed to observe the hydro-morphologic response of a laboratory flume to bed-load sediment transport and nonequilibrium upstream sediment feeding. The hydro-dynamic and the sediment feeding rates were kept constant in time. The temporal evolution of the longitudinal profiles of the stream bed and of the water surface was measured by imaging methods. The experimental data were used to (i) provide a phenomenological description of the aggradation process, recognizing different morphologic features superimposed on one another and (ii) characterize the height and velocity of propagation of aggrading sediment fronts. The front heights increased with increasing sediment supply, while the front celerity decreased. The celerity values were compared to a few predictors available in the literature. The literature formulae return celerity values scattered over almost two orders of magnitude, and present conflicting trends (a predicted celerity may either increase or decrease for increasing sediment feeding rate), thus stimulating further research on the topic.
\end{abstract}

\section{Introduction}

Flood events in mountain areas often involve the transport of large sediment volumes, generally produced by slope erosion or landslides. The consequent morphologic evolution of the river beds is of primary importance, because rapid flood events can induce significant aggradation/degradation of the bed. Sediment deposition increases the river bed level and, consequently, the maximum water levels, possibly causing overtopping; on the other hand, erosion near structures can make them unstable.

In this context, a key issue is the downstream migration of sediment for which a relatively scarce knowledge is available despite some experiments exist on propagation of sediment fronts [1-6]. Pioneering laboratory studies on aggradation were conducted by [1]; using uniform sand and different loading conditions, the authors proposed a predictor for the length of an aggradation front. A new version of this predictor was later provided by [2] after running a set of overloading experiments at a constant rate, in a recirculating flume. Similar experiments were performed by [3-5], while [6] considered the downstream migration of sediment pulses rather than stationary sediment feeding conditions. Predictors for the

\footnotetext{
* Corresponding author: alessio.radice@polimi.it
} 
downstream migration of sediment fronts were proposed by [3-4]. In a period of almost four decades of studies, the complexity of non-equilibrium flows has been hampering the assessment of reliable semi-empirical relations to predict the downstream migration of aggrading sediment fronts, calling for an improved phenomenological description of the process that could positively contribute to the development of prediction tools.

We performed an experimental study to observe the hydro-morphologic response of a laboratory flume subject to bed-load sediment transport and non-equilibrium upstream sediment feeding. Experimental control parameters include the bed slope, water discharge, sediment characteristics, upstream sediment feeding rate. The paper presents a series of runs with uniform lightweight sediment where both the hydro-dynamic and the sediment feeding rates were kept constant in time, the purpose of follow-up investigations being to extend the analysis to sediment migration under unsteady flows. The experimental results involve (i) the general shape of the river bed during the process, with a separation of morphologic processes taking place at different scales and (ii) a characterization of the height and velocity of propagation of aggrading and sediment fronts. The celerity of propagation of the fronts was finally compared to a few predictors that are available in the literature.

\section{Experimental set-up and procedure}

The physical modelling was performed in a rectangular tilting flume located in the Mountain Hydraulics Laboratory of the Politecnico di Milano. The flume (Fig. 1) is $5.2 \mathrm{~m}$ long, $0.3 \mathrm{~m}$ wide and $0.45 \mathrm{~m}$ deep. The channel is hinged at the inlet, and the longitudinal slope is adjusted by a screw jack; an additional support is present at the outlet to increase the system stability. Water is pumped from an underground sump to an upstream water tank, then flows into the flume and is discharged to a downstream tank, then to a recirculation channel and finally back into the sump. A tailwater regulation device (that is constituted by an array of sticks) is present at the downstream end of the flume. Sediment material can be fed at the flume inlet by a vibrating sediment hopper; the sediment transported by the flow is trapped at a flume outlet container, then removed, dried and poured back into the hopper. Polyvinyl Chloride (PVC) cylindrical particles (with size $=38 \mathrm{~mm}$, geometric standard deviation of the grain size distribution $=1.04$, density $=1443 \mathrm{~kg} / \mathrm{m}^{3}$, porosity $=0.45$ ) were used as the bedload sediment. A $0.15 \mathrm{~m}$ thick layer of loose particles was placed in the flume; in a $0.75 \mathrm{~m}$ long inlet reach, instead, the bed was fixed and composed of PVC particles glued onto acrylic plastic panels to avoid inlet scour.

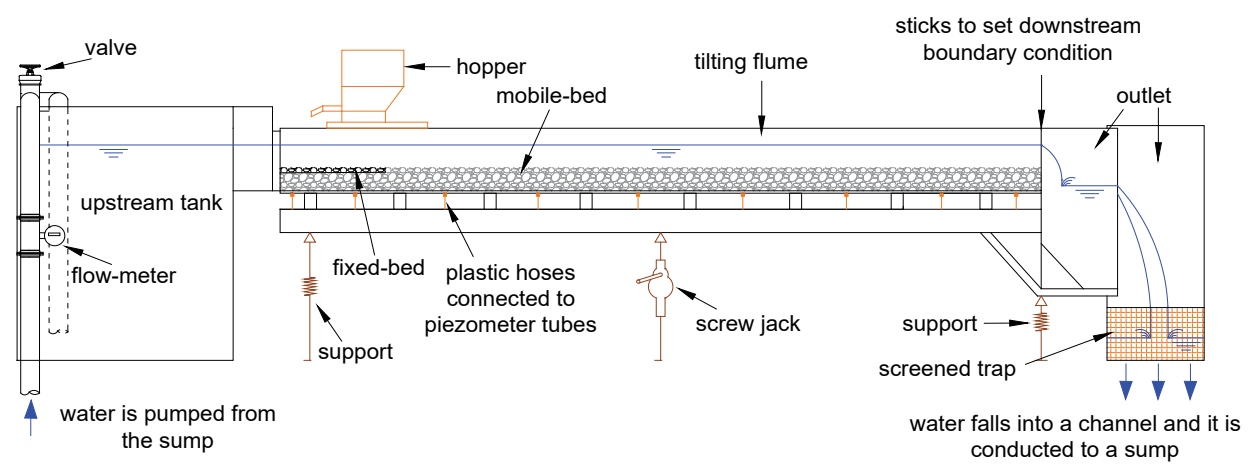

Fig. 1. Schematic diagram of the flume system. 
The water discharge was measured by an electro-magnetic flow meter, installed on a supply pipe. The sediment feeding rate was known from preliminary runs where a correspondence was determined between the hopper regulation (opening of a slot and vibration intensity) and the amount of fed sediment. Piezometric probes are attached to the channel at several stations, and all the piezometer pipes are close to each other on a wallanchored panel; the elevation of the water surface is measured from movies of the piezometer panel. A camera was placed beside the channel to capture in a single frame a view of the entire flume length; the longitudinal profile of the channel bed was measured from the acquired images. The used cameras were two Go-Pro Hero 4 Black Edition shooting 30 frames per second with a resolution of $1,920 \times 1,080$ pixel. Image frames were extracted from films every 10 seconds from the beginning of an experiment. Prior to delineation of bed profiles, a code for radial transformation of images [7] was applied to correct the barrel distortion due to the short lens mounted on the camera. Water surface and bed profiles were delineated from digital images manually.

Preliminary runs were performed to determined suitable tailwater regulations to impose quasi-uniform flows in the flume. Prior to execution of a run, the movable bed was scraped and the sediment was water-sprayed to avoid sediment lifting due to surface tension at water arrival. The flume was filled at a very low discharge (lower than $1 \mathrm{l} / \mathrm{s}$ ) to avoid sediment movement before the actual start of an experiment. A desired water elevation was reached with the low discharge used to fill the flume. Then, the flow rate was increased to a desired test value and, at the same time, some sticks were removed from the tailwater regulation device. The sediment hopper was switched on as soon as bed-load transport started to occur.

The experimental runs presented in this work are for a flume slope of $0.4 \%$, discharge of $14.7 \mathrm{l} / \mathrm{s}$, water depth of $0.12 \mathrm{~m}$ and different sediment feeding rates. Therefore, the present experiments represent a series with different overloading discharge starting from a quasiuniform flow condition, for which a sediment transport capacity returned by the equation of [8] was equal to $2.85 \times 10^{-5} \mathrm{~m}^{3} / \mathrm{s}$. Subcritical flow was always present, with practically no suspended load. The flow discharge, the stick arrangement at the downstream end and the sediment feeding rate were maintained constant during an aggradation test. Given a relatively low width-to-depth ratio (that was equal to 2.5), secondary currents could develop, that were however not investigated. Furthermore, preliminary experiments demonstrated a relatively well developed flow distribution in the channel, but this issue becomes irrelevant in the aggradation experiments because the fall of the fed sediment alters the flow distribution. The sediment feeding rates $\left(Q_{s}\right)$ and the run durations $(T)$ are furnished in Tab. 1.

Table 1. Characteristics of the aggradation experiments.

\begin{tabular}{|c|c|c|c|c|}
\hline Experiment & AE1 & AE2 & AE3 & AE4 \\
\hline$O_{s}\left(\mathrm{~m}^{3} / \mathrm{s}\right)$ & $5.22 \times 10^{-5}$ & $4.31 \times 10^{-5}$ & $3.37 \times 10^{-5}$ & $4.31 \times 10^{-5}$ \\
\hline$T(\mathrm{~min})$ & 17.2 & 32.1 & 25.4 & 35.5 \\
\hline
\end{tabular}

\section{Results}

\subsection{Phenomenological description of aggradation profiles}

Figure 2 shows the evolution of the water and bed surface (in terms of $z$ - $z_{\text {bed }}$, meaning that the origin of the $z$ axis is set at the initial elevation of the sediment bed) for experiment AE1 (similar graphs of spatial and temporal evolution of the sediment front were produced for all the other tests, illustrating analogous characteristics). The deposition of particles starts at the upstream end, and a reach where sediment aggradation occurs increases progressively in 
length while the sediment is fed into the channel. The shape of the measured longitudinal bed profiles presents oscillations that indicate the presence of bed-forms superimposed to the deposition front. These morphological features are qualitatively comparable with those observed in the flume aggradation experiments of [4]. Bed-forms did not only develop on the deposition front, but also in the downstream portion of the flume, triggered by the local bedload transport conditions. The water level obviously reacted to the modified bed conditions, assuming lower elevations above the deposition front (consistently with the subcritical approaching flow conditions).

The time evolution of the bed at five different locations along the channel is depicted for experiment AE1 in Fig. 3, that is a counterpart of Fig. 2. The bed elevation increases first in the upstream locations, then the deposition front reaches further locations along the flow. Also the plots of the bed elevation in time reveal the presence of bed-forms superimposed to the aggradation front, as well as the adaptation of the free surface to the modified channel geometry. These variations of the bed and water surface are similar to those described by [3].

The dashed lines in Fig. 2 and 3 identify the propagation of the sediment aggradation front. The slope of these lines qualitatively resembles a celerity of propagation that will be analysed in detail in the following section of the manuscript.
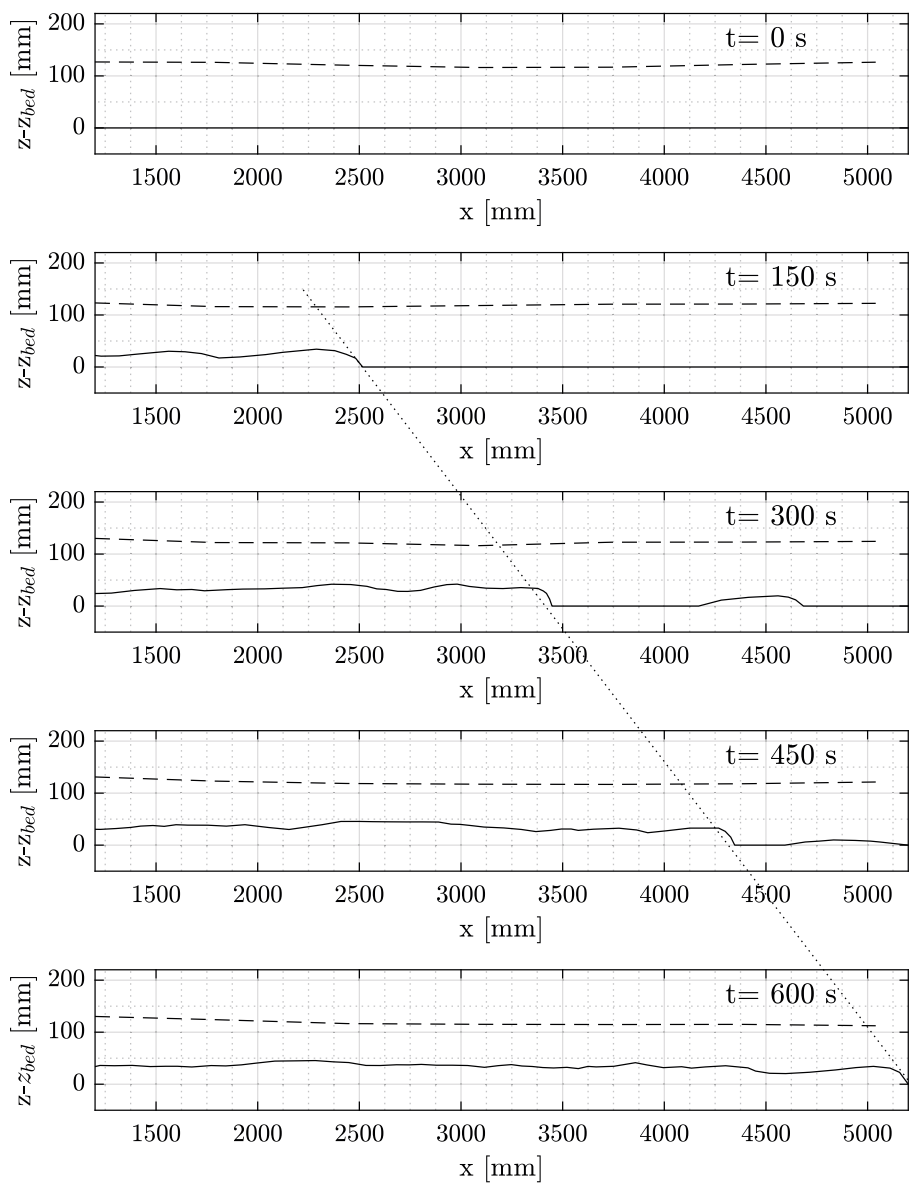

Fig. 2. Spatial evolution of bed and water profiles for experiment AE1 and different times. The $x$ axis corresponds to the stream-wise distance from the inlet. The variable $z_{b e d}$ corresponds to the bed elevation at any position before the arrival of the aggradation front. The bed profile is represented in solid line while the water profile is drawn in dashed line. 

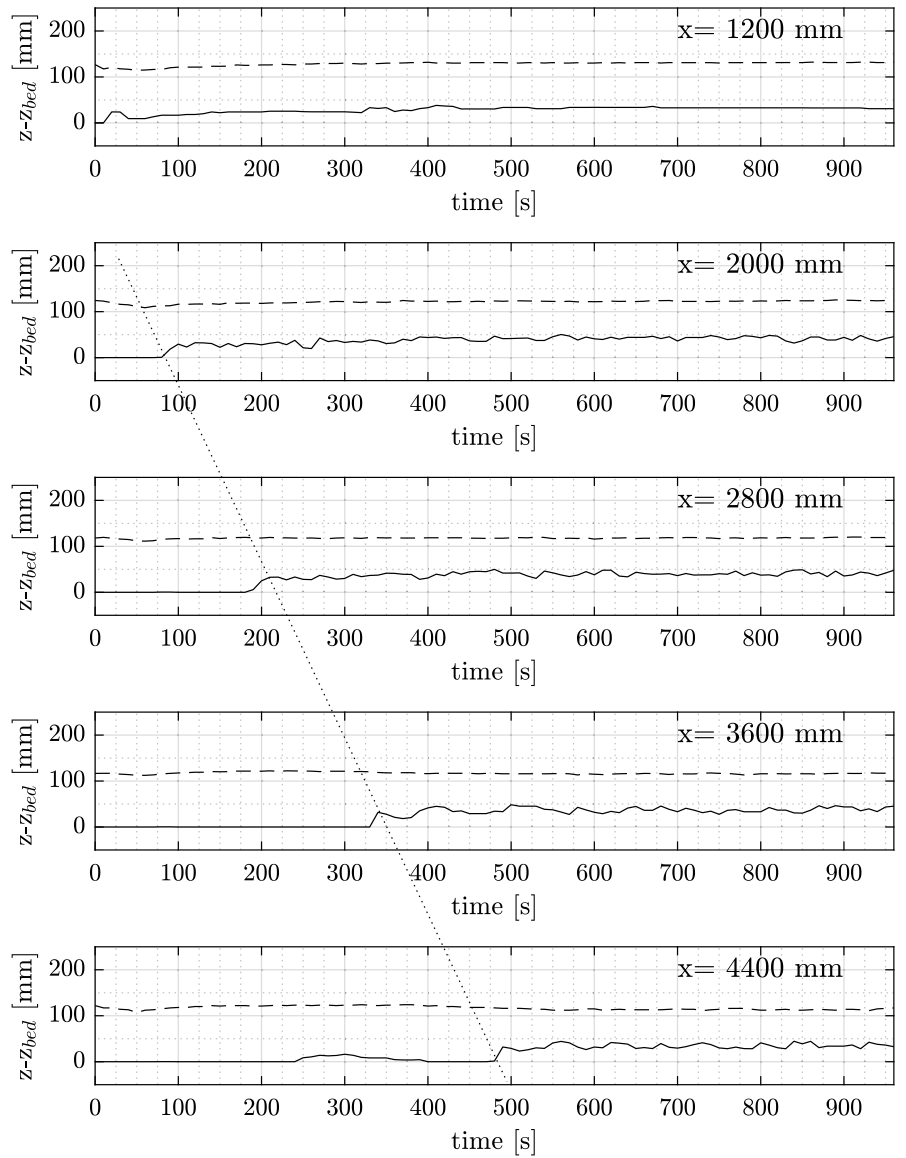

Fig. 3. Temporal evolution of bed and water profiles for experiment AE1 and different locations. The bed profile is represented in solid line while the water profile is drawn in dashed line.

\subsection{Front properties: height and celerity}

An average height was computed for the sediment fronts and is shown in Fig. 4(a), presenting heights that increase with increasing $Q_{s}$.

The front celerity $c_{w}$ was determined identifying the instants of front arrival at different locations, using plots like those in Fig. 3. The identification of instants was preferred to the identification of locations from plots like those in Fig. 2, because spatial trends were sometimes more irregular than temporal ones (for example, [4] identified the sediment fronts from spatial profiles but had to use spline interpolation to regularize them). The propagation of the sediment fronts for the present experiments is depicted in Fig. 4(b). Based on a simplifying assumption that the celerity remained constant during the aggradation process, it was assessed as the slope of a linear regression of the points in Fig. 4(b).

The variation of the front celerity with different sediment feeding rates is presented in Fig. 4(c). The front celerity was found to decrease for increasing sediment feeding rate. Since the present experiments were run with the same reference hydro-dynamic conditions, a decreasing trend of $c_{w}$ with $Q_{s}$ indicates that a prescribed flow can move faster lower interfering sediment volumes, and vice versa. This behaviour can be reasonably interpreted if one considers the extreme case of a very large sediment feeding, that would be assumed to totally obstruct the flume section and create something like an earth dam (leading, therefore, 
to $\left.c_{w}=0\right)$. On the other side, with a sediment feeding rate equal to the transport capacity of the flow (in other words, for excess sediment feeding rate equal to 0 ), no front will be observed, which can be interpreted as an infinite celerity.

(a)

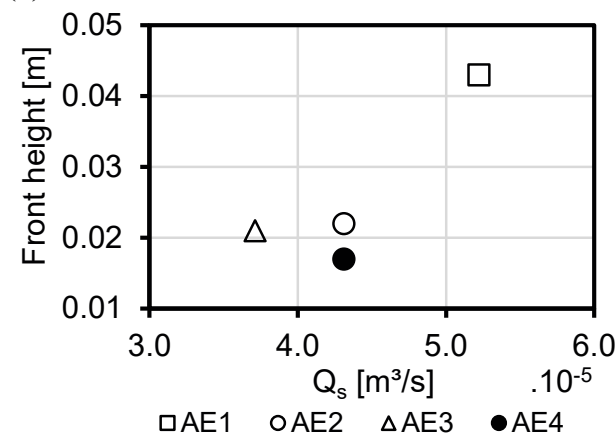

(c)

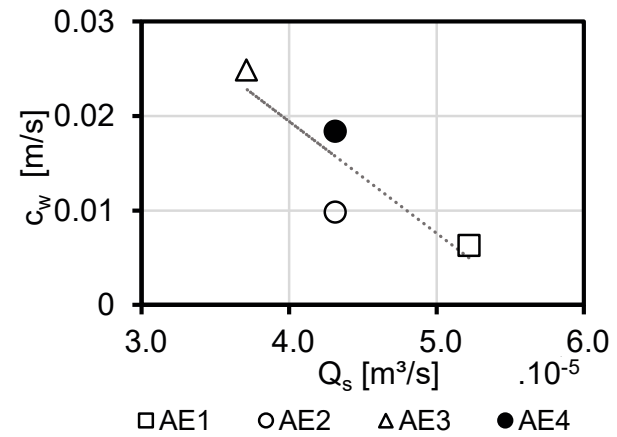

(b)

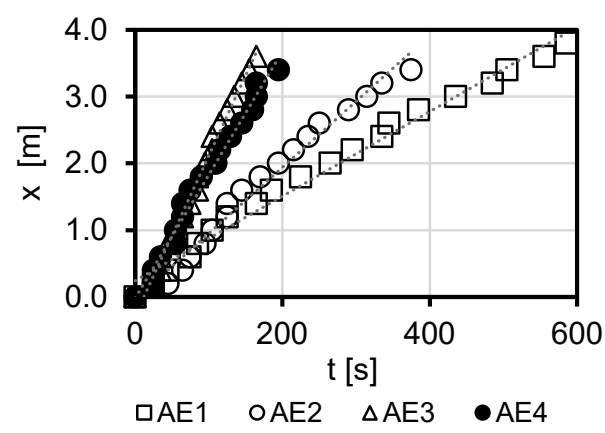

(d)

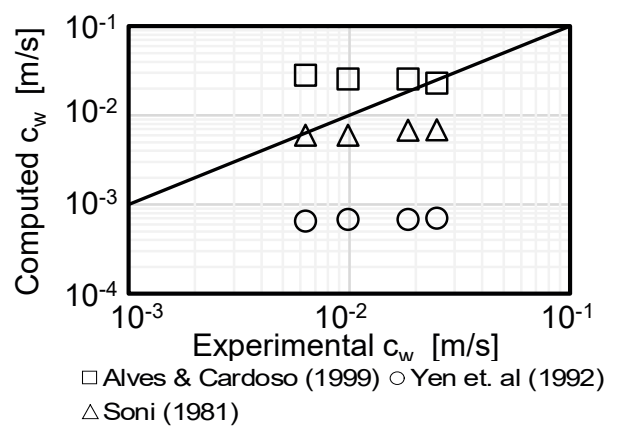

Fig. 4. Deposition front properties: (a) sediment front height versus sediment feeding rates (b) temporal progression of the sediment fronts; (c) front celerity versus sediment feeding rate; (d) comparison between computed and experimental celerity values, with the solid line as the identity line.

The celerity values determined experimentally were compared to those predicted by the formulae proposed by $[1,3,4]$. A constant value of celerity is returned by the formulae by $[4,5]$, leading to an immediate comparison with the experimental values determined for the present runs. The equation by [1], instead, furnishes the length of the aggradation front as a function of time, with a time-varying front celerity. In order to apply the formula by [1], a time was computed for the arrival time of the sediment front to the downstream end of the flume and a mean celerity was computed accordingly. Figure 4(d) presents the outcome of the comparison. The values computed from the literature are scattered within two orders of magnitude. The formulae by $[1,3]$ predict a trend that is consistent with the experimental one (the computed celerity increases when the measured one increases), while the predictor of [5] returns an opposite trend. In all the cases, the variability of the computed values is much less than that of the measured values. The equations of $[1,4]$ are more in agreement with the present data than the formula by [3], even though no equation fits the data in the entire experimental range. For our experiment with the largest sediment feeding rate the equation of [1] performs better, while for the experiment with the lowest sediment feeding rate a good prediction is obtained using the formula by [4]. 


\section{Conclusions}

Aggradation induced by upstream sediment feeding was studied experimentally. Laboratory tests were performed in a narrow flume to analyse the impact of variable sediment overloading discharge on the propagation of an aggradation front. The evolution of the bed and water longitudinal profiles was obtained from digital images taken during the experiments, allowing the measurement of depositional front height and celerity. Experimental celerity values were then compared to literature predictors. As a result, and for the covered experimental range, the following conclusions can be stated:

0 the bed elevation gradually increases due to the appearance of a deposition front that migrates downstream as the process develops.

- The bed profiles depict the presence of bed-forms superimposed to the deposition front.

- The height of sediment front increases for increasing sediment feeding rate.

- Increasing values of sediment feeding rate lead to a decrease in the celerity of the aggradation front.

- The predictive ability of literature formulae for the celerity of aggradation fronts seems still unsatisfactory, stimulating further research on the topic.

The goal of the present study is to provide insight on the propagation of sediment fronts. However, the similarity between our experiments and Saint-Venant-, Exner-based numerical simulations of river morphologic processes is also evident. in fact, a hydro-morphologic model rarely involves the entire river length; most frequently, only a reach under specific analysis is considered and a sediment supply is input at the upstream boundary to mimic the interface between the computed river reach and the remaining part of the catchments. The supplied material then migrates downstream. Therefore, the experimental conditions used in this study are relevant benchmark situations for numerical modelling of river hydromorphologic processes.

We acknowledge financial support by Fondazione Cariplo through the project entitled Sustainable MAnagement of sediment transpoRT in responSE to climate change conDitions (SMART-SED).

\section{References}

1. J.P. Soni, K.G. Ranga Raju, R.J. Garde, J. Hydraul. Eng., 106 (1980)

2. J.P. Soni, J. Hydrol., 49 (1981)

3. C.L. Yen, S.Y. Chang, H.Y. Lee, J. Hydraul. Eng., 118 (1992)

4. E. Alves, A.H. Cardoso, Int. J. Sed. Res., 14 (1999)

5. A. Miglio, R. Gaudio, F. Calomino, J. Hydro-Environ. Res., 3 (2009)

6. L.S. Sklar, J. Fadde, J.G. Venditti, P. Nelson, M.A. Wydzga, Y. Cui, W.E.Dietrich, Water Resour. Res., 45 (2009)

7. A. Radice, R. Aleixo, S.A. Hosseini Sadabadi, S. Sankar, Proc. of HydroSenSoft 2017, Madrid, Spain (2017)

8. E. Meyer-Peter, R. Müller, Proc. of the 2nd meeting of IAHR, Stockholm, Sweden (1948) 